\title{
Long-read transcriptome sequencing provides insight into lignan biosynthesis during fruit development in Schisandra chinensis
}

\author{
Chang Pyo Hong ${ }^{1 *}$, Chang-Kug Kim², Dong Jin Lee ${ }^{1}$, Hee Jeong Jeong ${ }^{3}$, Yi Lee ${ }^{3}$, Sin-Gi Park ${ }^{1}$, Hyo-Jin Kim${ }^{4}$, \\ Ji-Nam Kang ${ }^{2}$, Hojin Ryu ${ }^{5}$, Soo-Jin Kwon ${ }^{2}$ and Sang-Ho Kang ${ }^{2^{*}}$
}

\begin{abstract}
Background: Schisandra chinensis, an ancient member of the most basal angiosperm lineage which is known as the ANITA, is a fruit-bearing vine with the pharmacological effects of a multidrug system, such as antioxidant, anti-inflammatory, cardioprotective, neuroprotective, anti-osteoporosis effects. Its major bioactive compound is represented by lignans such as schisandrin. Molecular characterization of lignan biosynthesis in S. chinensis is of great importance for improving the production of this class of active compound. However, the biosynthetic mechanism of schisandrin remains largely unknown.
\end{abstract}

Results: To understand the potential key catalytic steps and their regulation of schisandrin biosynthesis, we generated genome-wide transcriptome data from three different tissues of S. chinensis cultivar Cheongsoon, including leaf, root, and fruit, via long- and short-read sequencing technologies. A total of 132,856 assembled transcripts were generated with an average length of $1.9 \mathrm{~kb}$ and high assembly completeness. Overall, our data presented effective, accurate gene annotation in the prediction of functional pathways. In particular, the annotation revealed the abundance of transcripts related to phenylpropanoid biosynthesis. Remarkably, transcriptome profiling during fruit development of S. chinensis cultivar Cheongsoon revealed that the phenylpropanoid biosynthetic pathway, specific to coniferyl alcohol biosynthesis, showed a tendency to be upregulated at the postfruit development stage. Further the analysis also revealed that the pathway forms a transcriptional network with fruit ripening-related genes, especially the ABA signaling-related pathway. Finally, candidate unigenes homologous to isoeugenol synthase 1 (IGS1) and dirigent-like protein $(D / R)$, which are subsequently activated by phenylpropanoid biosynthesis and thus catalyze key upstream steps in schisandrin biosynthesis, were identified. Their expression was increased at the postfruit development stage, suggesting that they may be involved in the regulation of schisandrin biosynthesis in S. chinensis.

Conclusions: Our results provide new insights into the production and accumulation of schisandrin in S. chinensis berries and will be utilized as a valuable transcriptomic resource for improving the schisandrin content.

Keywords: Fruit development, Lignan biosynthesis, Long-read transcriptome sequencing, Phenylpropanoid biosynthesis, Schisandra chinensis

\footnotetext{
*Correspondence: changpyo.hong@theragenbio.com; hosang93@korea.kr

${ }^{1}$ Theragen Bio Co., Ltd., Suwon 16229, Republic of Korea

${ }^{2}$ Genomics Division, National Institute of Agricultural Sciences, RDA,

Jeonju 54874, Republic of Korea

Full list of author information is available at the end of the article
}

\begin{abstract}
Background
Schisandra chinensis, also known as 'Omija' (Korea), 'Wuweizi' (China), or 'Gomishi' (Japan), is a climbing species with a generation time of five years that belongs to the order Austrobaileyales, which consists of earlydiverging angiosperms [1]. The natural habitats of $S$.
\end{abstract}


chinensis are mostly within northeastern Asia; they show a uniform distribution of genetic diversity and low genetic differentiation because of extensive gene flow [2]. The berry fruits of $S$. chinensis are clustered in grape-like bunches and exhibit five flavors: salty, sweet, sour, pungent (spicy), and bitter. The fruits contain many bioactive compounds, including lignans, triterpenes, phenolic acids, flavonoids, essential oils, and polysaccharides [3, 4], and they have been used as an important traditional medicine in northeastern Asia. In particular, dibenzocyclooctadiene lignans, including schisandrin and gomisin, present in S. chinensis fruit extracts exhibit pharmacological effects such as antitumor, anti-inflammatory, antioxidative, hepatoprotective, antihypertensive, and anti-osteoporosis activities [3-7]. Moreover, fruits have the potential to effectively protect against neuronal cell damage and to significantly enhance cognitive performance, suggesting their usefulness as new therapeutic agents for treating neurodegenerative diseases [8].

In general, biosynthesis of lignans in plants is linked to phenylpropanoid pathway in an upstream function and branches after synthesis of coniferyl alcohol. A succession of specific steps, involving in catalytic reactions of dirigent (DIR), pinoresinol-lariciresinol reductase (PLR), secoisolariciresinol dehydrogenase (SILD), O-methyltransferases (OMTs; i.e. OMT1 and OMT3), cytochrome P450 families (CYPs; i.e. CYP719A23, CYP71CU1, CYP71BE54, CYP82D61), and UDP-glucose-dependent glucosyltransferases (UGTs) [9-15], lead to production of lignans (Additional file 4: Fig. S7). The specific steps is closely related to the production of $(-)-4^{\prime}$-desmethylepipodophyllotoxin, an etoposide aglycone, that was well characterized in Podophyllum hexandrum [14]. Interestingly, the genetic engineering of lignan biosynthetic enzyme genes (i.e. PLR-RNAi in Forsythia) demonstrated the direct alteration of the lignan production in plants [16]. Unlike podophyllotoxin biosynthetic pathway that starts with the synthesis of pinoresinol in lignan branch, dibenzocyclooctadiene lignan is initiated with the synthesis of isoeugenol [17]. In early steps for the biosynthesis of dibenzocyclooctadiene lignan, isoeugenol molecules are further metabolized to verrucosin and dihydroguaiaretic acid by catalytic reactions of DIR and PLR, respectively [17]. The resulting dihydroguaiaretic acid is converted to schisandrin or gomisin via several steps that are catalyzed by putative OMTs, CYPs and/ or UGTs. However, the fully understanding of this biosynthetic pathway is still far from complete. Because schisandrin biosynthesis is distinct from podophyllotoxin biosynthetic pathway, it may be limited to understand schisandrin biosynthesis in S. chinensis based on podophyllotoxin biosynthetic pathway. Thus, prior to discovering key active enzymes related to schisandrin biosynthesis, the study of the regulation of genes, such as $D I R, P L R$, and IGS1, involved in early step of the biosynthetic pathway can provide insight into the production and accumulation of schisandrin.

The accumulation of polyphenolic compounds such as lignans is reported to be affected by fruit maturation stages, which are dependent on environmental conditions and the genetic makeup of a species $[18,19]$. Fruits undergo various physiological and biochemical changes during maturation, consequently altering their bioactive composition. Although very little research has been conducted to investigate the changes in nutraceutical components during ripening in S. chinensis, the contents of lignans in the fruits of $S$. chinensis have been studied. Previous studies have reported high contents of schisandrin in the ripened fruits of $S$. chinensis [20,21]. Szopa et al. [6] identified the highest contents of gomisin N, followed by schisandrin $\mathrm{A}$, schisandrin, gomisin $\mathrm{D}$, schisantherin $\mathrm{B}$, gomisin $\mathrm{A}$, angeloylgomisin $\mathrm{H}$ and gomisin J. In connection with the fruit ripening and the accumulation of polyphenols, the phenylpropanoid biosynthesis is known to activate their production and accumulation [22, 23], probably providing a clue that explains how schisandrin is synthesized by the activation of phenylpropanoid biosynthesis. Therefore, such information is useful for optimizing the harvesting time of a fruit species to achieve the maximum nutraceutical potential and provides insight for designing new strategies to improve the production of clinically effective components in S. chinensis.

Long-read transcriptome sequencing, such as isoform sequencing (Iso-Seq) via the single-molecule real-time (SMRT) sequencing, produces longer and more accurate transcripts with a high level of assembly completeness and gene annotation [24]. Thus, the sequencing approach has been recently utilized for functional genomics in large complex or uncharacterized plant species [24-26]. For example, Wang et al. [25] reported that maize yielded considerable nonredundant full-length transcript isoforms, corresponding to approximately 26,946 genes. In addition, the genome coverage of Iso-Seq data is near saturation with maximum transcriptome diversity. Thus, de novo transcriptome assembly using Iso-Seq will help in the exploration of genes related to the biosynthesis of major bioactive compounds in medicinal plants with no reference genome, and will facilitate understanding of the underlying mechanism that provides new insight to the physiological role of these metabolites and their functional diversity in plants. Ultimately, long-read transcriptome sequencing may be a cost-effective gold standard for building reference gene sets for conducting functional genomics in plants.

To obtain a more comprehensive view of the transcriptome in S. chinensis, especially insight into the regulation 
of lignan biosynthesis during fruit development, we generated de novo transcriptome data derived from three different tissues, including leaves, roots, and fruits, via long-read transcriptome sequencing coupled with RNASeq. Although the genomic information of S. chinensis is not yet sufficient, our data allowed highly accurate gene annotation with ORF prediction, thus being helpful for identifying candidate genes involved in lignan biosynthesis. Furthermore, we investigated the transcriptional changes of these genes during fruit development in $S$. chinensis. In particular, the analysis revealed a transcriptional network between phenylpropanoid biosynthesis and ripening-related phytohormones. Our data can be used as a valuable transcriptomic resource for discovering genes associated with the production of medicinal compounds in S. chinensis and are helpful for understanding the regulation of lignan biosynthesis in its fruit.

\section{Results}

De novo transcriptome assembly in S. chinensis via longand short-read sequencing technologies

Using PacBio Iso-Seq and llumina RNA-Seq, we generated de novo transcriptome assembly in a S. chinensis cultivar Cheongsoon that shows the characteristics of large fruits, high yielding ability, and tolerance to brown leaf spot or powdery mildew compared with a wild type Sobaeksan (Additional file 5; Table S4). We first generated large cDNA fragments from $S$. chinensis by using Iso-Seq (Fig. 1a), which favors the reverse transcription of intact, full-length mRNA molecules and identifies candidate genes related to interesting functional pathways, such as phenylpropanoid and lignan biosynthesis. cDNA libraries were size-selected for lengths of $1-2 \mathrm{~kb}, 2-3 \mathrm{~kb}, 3-6 \mathrm{~kb}$, and $>6 \mathrm{~kb}$ from RNA samples of $S$. chinensis pooled from three tissues (leaves, roots, and fruits) and sequenced on the PacBio RS II platform. A total of 9.5 million subreads were generated (Additional file 1: Table S1) and merged into 51,297 to 79,222 isoform clusters (Additional file 3: Table S3). After consensus calling, quality filtration, and the removal of transposable element (TE) sequences, the sequences were clustered into a total of 92,378 assembled transcripts, referred to as unigenes. Furthermore, to cover small transcripts, especially those of less than approximately $1 \mathrm{~kb}$, which may be missed by size selection in Iso-Seq library construction, a total of 107,748

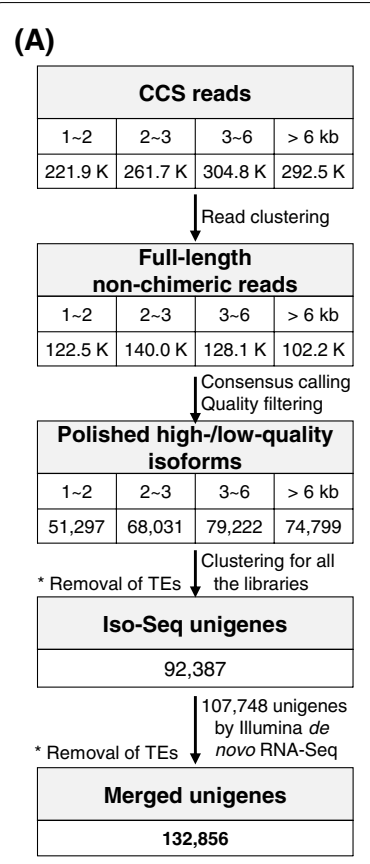

(B)

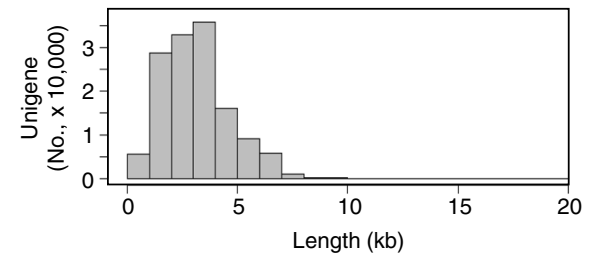

(D)

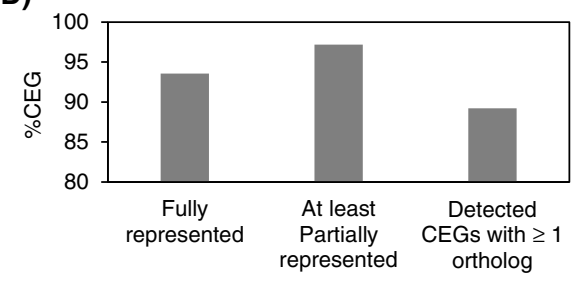

(C)

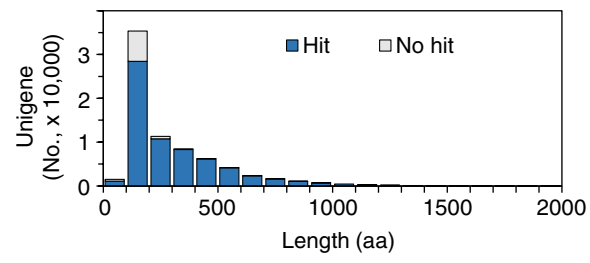

(E)

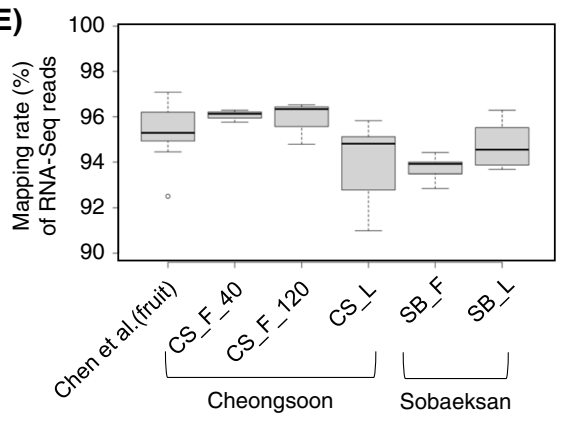

Fig. 1 Overview of transcript assembly in S. chinensis. a. Procedure of transcript assembly for S. chinensis. Clean subreads of each size class (1 2kb, $2 \sim 3 \mathrm{~kb}, 3 \sim 6 \mathrm{~kb}$, and $>6 \mathrm{~kb}$ ) were merged into the corresponding isoform cluster and classified as full-length (FL) and/or non-FL reads. After consensus sequence calling and quality filtration were carried out, the sequences were clustered into Iso-Seq unigenes. Unigenes derived from the Iso-Seq and Illumina de novo RNA-Seq procedures were merged after TE sequences were removed. $\mathbf{b}$. The length distribution of unigenes. $\mathbf{c}$. The length distribution of translated sequences (aa) predicted from unigenes. $\mathbf{d}$. CEGMA evaluation. The completeness (\%) of the unigenes ('fully represented (EGs') was assessed by CEGMA. e. Mapping of RNA-Seq samples derived from fruit and leaf tissues. In the figure, RNA-Seq data of four fruit, including Chen et al. [27] (from Jilin, China), CS_F_40 and CS_F_120 (sampled at 40 and 120 DAFs of fruit in Cheongsoon), and SB_F (Sobaeksan), and two leaf samples, including CS_L (Cheongsoon) and SB_L (Sobaeksan), were analyzed 
unigenes (N50 of $429 \mathrm{bp}$ ) that were de novo assembled using Illumina RNA-Seq data derived from fruit tissue were merged into Iso-Seq unigenes. A total of 132,856 unigenes were finally obtained with a cumulative length of approximately $246.9 \mathrm{Mb}$. This procedure is summarized in Fig. 1a. The average length of the unigenes was $1.9 \mathrm{~kb}$, with a distribution of $2 \sim 4 \mathrm{~kb}$ (Fig. 1b); this average length was longer than that previously reported by Chen et al. [27] of $754 \mathrm{bp}$. Open reading frames (ORFs) were predicted in 73,774 unigenes (55.5\%), accounting for a cumulative length of approximately $63.9 \mathrm{Mb}$, with an average length of 288 aa (Fig. 1c). Remarkably, 56,038 of these unigenes were predicted to contain complete full-length ORFs, most of which were generated from Iso-Seq (Additional file 4: Fig. S1).

We assessed the quality of the S. chinensis unigenes. First, the Core Eukaryotic Genes Mapping Approach (CEGMA), which assesses a highly reliable set of gene annotations in genome and transcriptome assemblies, revealed a completeness of $93.6 \%$ (Fig. 1d). Second, various RNA-Seq samples that were generated from leaves and fruits of Cheongsoon and Sobaeksan were mapped to the unigene dataset with coverage ranging from 91.0 to $97.1 \%$ (Fig. 1e), showing high data mapping coverage. Therefore, our data indicate the high quality of the assembly and mapping coverage of the reads for $S$. chinensis, which is a helpful transcriptome reference dataset.

\section{Functional annotation of unigenes}

All unigenes were annotated using homology-based searches. Of all unigenes, 90,930 unigenes (68.4\%) showed significant homology with protein sequences in the UniProt (69,782 hits), TAIR (78,952 hits), NCBI NR $(87,851$ hits), and InterPro (46,222 hits) databases. In the searches, 83,228 (91.5\%) annotated unigenes were predicted to be known genes based on UniProt and TAIR, which accept primary sequences derived from sequencing experiments with functional information, showing good annotation quality. In particular, 39,776 of the known unigenes were predicted to be known genes based on the identification of protein domains (Fig. 2a), showing outstanding hit scores. Of the annotated unigenes, 75,748 were identified to be derived from Iso-Seq data, showing efficient transcript annotation by long-read sequencing. We also examined unigenes homologous to known transcription factor (TF) sequences by searching against PlantTFDB using BLASTX with a cutoff of $1 E-05$. A total of 21,800 unigenes classified into $58 \mathrm{TF}$ families were identified. In the search, the bHLH, NAC, WRKY, MYB/-related, FAR1, C3H, B3, C2H2, ERF, GRAS, bZIP, HB-other,

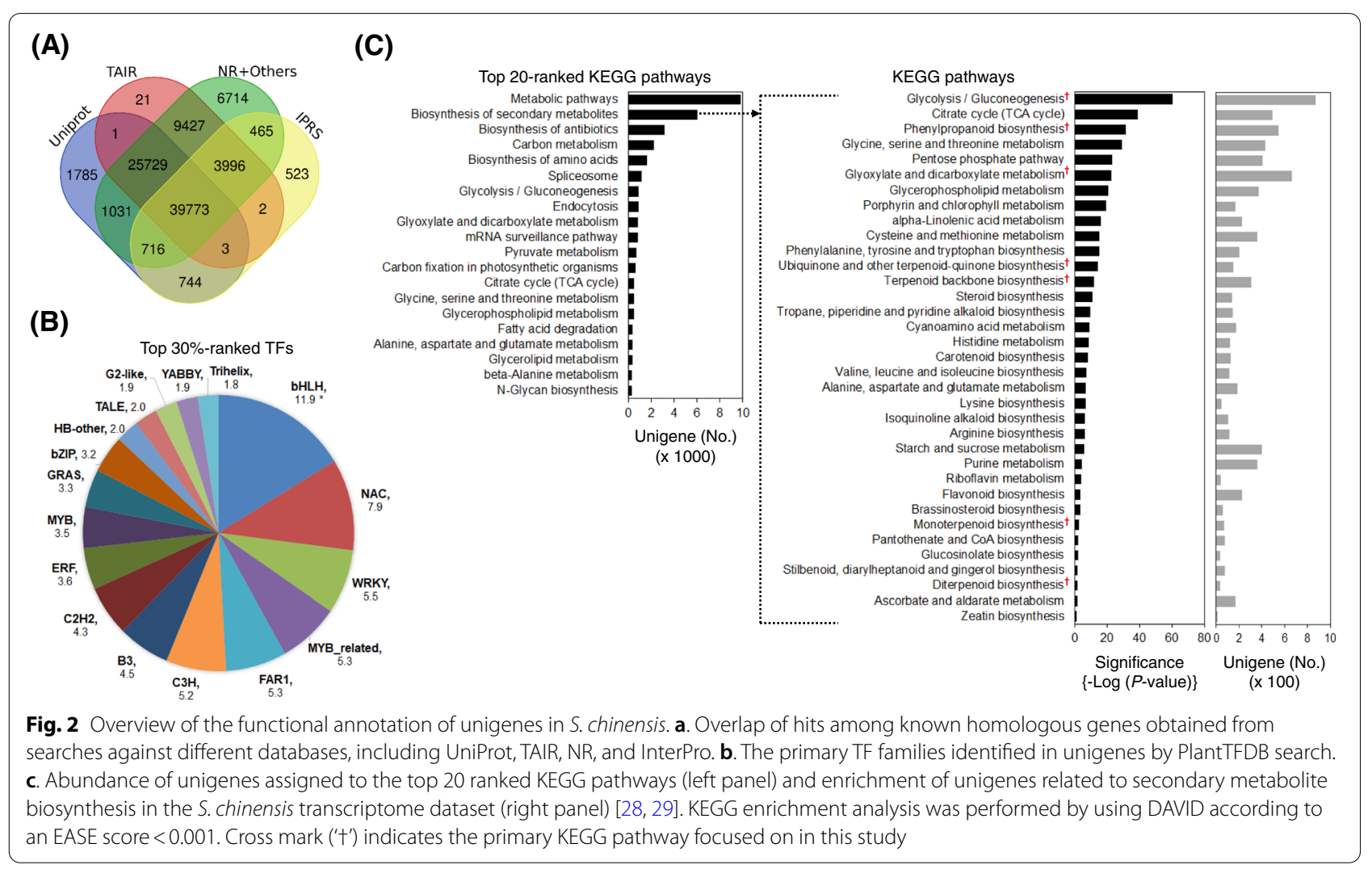


TALE, G2-like, YABBY, and Trihelix families were abundant in S. chinensis, making up the top $30 \%$ of all families according to abundance (Fig. 2b). Interestingly, bHLH, NAC, MYB, ERF, GRAS, bZIP, and TALE are involved in fruit development and/or plant growth [30]. The predominant proteins in terms of Pfam domains included P-loop-containing nucleotide triphosphate hydrolase $(5.4 \%)$, various protein kinases $(17.1 \%)$, tetraand penta-tricopeptides (5.7\%), and NAD(P)-binding protein $(2.1 \%)$ (Additional file 4: Fig. S2a). In addition, UniProtKB keywords associated with functional membranes $(7 \%)$, alternative splicing (3.7\%), transferases (3.7\%), the nucleus $(3.6 \%)$, and binding (6.2\%) were highly abundant (Additional file 4: Fig. S2b).

To further predict and classify unigene functions, the annotated unigenes were analyzed according to KEGG pathway assignments. A total of 12,940 unigenes were assigned to 20 primary pathways. The most common pathways were 'metabolic pathways', 'biosynthesis of secondary metabolites', 'biosynthesis of antibiotics', 'carbon metabolism,' 'terpenoid biosynthesis', and 'biosynthesis of amino acids' (left panel in Fig. 2c). In particular, the enrichment analysis for transcripts related to 'biosynthesis of secondary metabolites' revealed the significance of pathways related to 'glycolysis/gluconeogenesis', 'TCA cycle', 'glyoxylate and dicarboxylate metabolism', 'phenylpropanoid biosynthesis', 'amino acid-related metabolism', 'lipid-related metabolism', 'terpenoid biosynthesis', and 'carotenoid biosynthesis' (right panel in Fig. 2c). Interestingly, a high abundance of transcripts related to phenylpropanoid biosynthesis was identified. This result seems to suggest that the activation of the phenylpropanoid pathway in S. chinensis is associated with the biosynthesis of polyphenols such as lignans or flavonoids [22, 23]. Our data show that long-read-based transcriptome sequencing resulted in more effective, accurate annotation or prediction for elucidating genes associated with interesting traits or functional pathways in S. chinensis.

\section{Characterization of transcripts in phenylpropanoid and lignan biosynthesis}

In relation to the lignan biosynthetic pathway, in the podophyllotoxin pathway, especially the pathway leading to etoposide aglycone, (-)-4'-desmethylepi podophyllotoxin, an identified biosynthetic gene, has been studied in detail [14]. However, the biosynthetic mechanism of schisandrin production remains largely unknown, and only early steps for catalyzing the synthesis of dihydroguaiaretic acid that is converted to the dibenzocyclooctadiene skeleton have been studied [31]. Nevertheless, to understand lignan biosynthesis in S. chinensis, we identified transcripts related to lignan biosynthetic pathways for deoxypodophyllotoxin and dihydroguaiaretic acid and the phenylpropanoid biosynthetic pathway for coniferyl alcohol, which is utilized for activating the lignan biosynthetic pathway (Fig. 3b). For phenylpropanoid biosynthesis, a total of 61

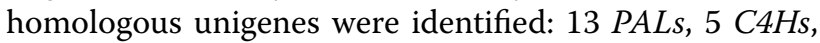
9 4CLs, 14 HCTs, 4 C3Hs, 5 CCoAOMTs, 3 CCRs, and $8 C A D s$ (Fig. 3a). For lignan biosynthesis, 76 homologous unigenes were also identified: 4 IGSs, 9 DIRs, 4 PLRs, 11 SILDs, 8 OMT1/3s-like, 31 CYP71CU1s-like, 6 CYP719A23/24s-like, and 3 2-ODDs (Fig. 3a and b). This result shows the conservation of those biosynthetic pathways in S. chinensis. Of those unigenes identified, unigenes homologous to $P A L, H C T, 4 C L$ and $C A D$ for phenylpropanoid biosynthesis and DIR, SILD, OMT1/3, and CYP71CU1 for lignan biosynthesis were relatively abundant (Fig. 3a and b).

Among the identified unigenes, full-length ORFs homologous to IGS, DIR and PLR, which essentially contribute to the early step in the biosynthesis of the two lignans, were identified by analyzing conserved protein domains through multiple-sequence alignment with previously reported target genes (Fig. 3c). First, four IGS1 unigenes, KSC_ISO-074408, - 090301, - 004209 and-08352, which were distinct from eugenol synthase 1 (EGS1) or isoflavone reductase, were identified

\footnotetext{
(See figure on next page.)

Fig. 3 Identification of candidate genes related to phenylpropanoid and lignan biosynthetic pathways in S. chinensis. a. Identification of unigenes homologous to genes related to these two biosynthetic pathways. $\mathbf{b}$. Expression of candidate unigenes in the two pathways in leaf and fruit tissues. After quantifying gene expression from RNA-Seq data derived from the leaves and fruits of S. chinensis (Cheongsoon) with five replicates, the $\log _{2}$-scale fold changes of each of gene between leaf and fruit were calculated. In Figure, each small square cell indicates unigene homologous to gene encoding enzyme involved in the pathways. In addition, expression change of each unigene between leaf and fruit is shown with heatmap. Mark' $x$ ' in small square cell indicates no unigene. c. Multiple-sequence alignment of DIR, IGSI, and PLR homologs. Sequence conservation was identified among targeted genes (UniProt accession number: Q9SDR7 for DIR, Q15G13 for IGS1, and O65679 for PLR), the corresponding primary protein domains (dirigent for DIR, PCBER_SDR_a for IGSI, and NmrA-like family for PLR), and the corresponding homologous unigenes. d. Phylogeny of IGS1 homologs on the basis of the relationships among major linages of angiosperms sequenced, including basal angiosperms (GenBank accession number: XP_006859191.1 and XP_020532181.1 Amborella trichopoda and RWR83219.1 and RWR85316.1 for Cinnamomum micranthum), rosids (XP_021900462.1 for Carica papaya, XP_007022888.1 for Theobroma cacao, XP_007213116.1 and XP_007212642.2 for Prunus persica, XP_002310455.1 for Populus trichocarpa, and RVX21189.1 for Vitis vinifera), asterids (Q15G13.1 for Petunia x hybryda and CAA2957992.1 and CAA2959183.1 for Olea europaea) reported by the Amborella Genome Project [32]. e. Phylogenetic analysis between IGS1 and PLR homologs via the maximum likelihood-based method
} 


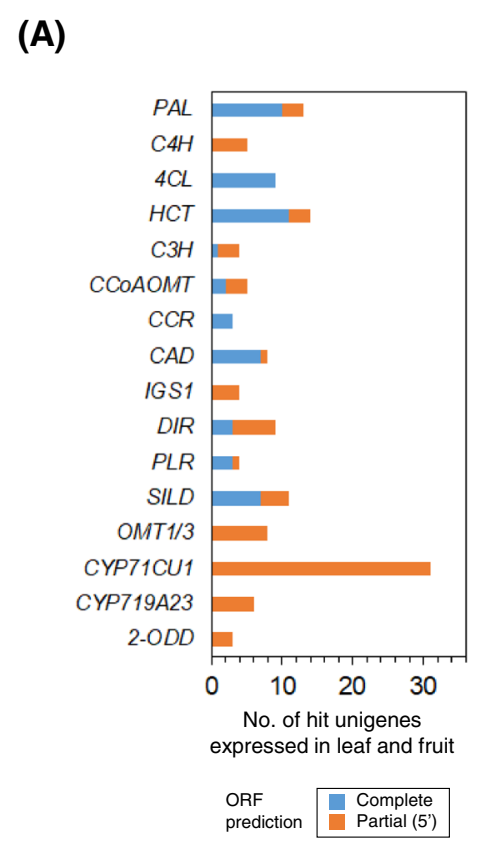

(B)

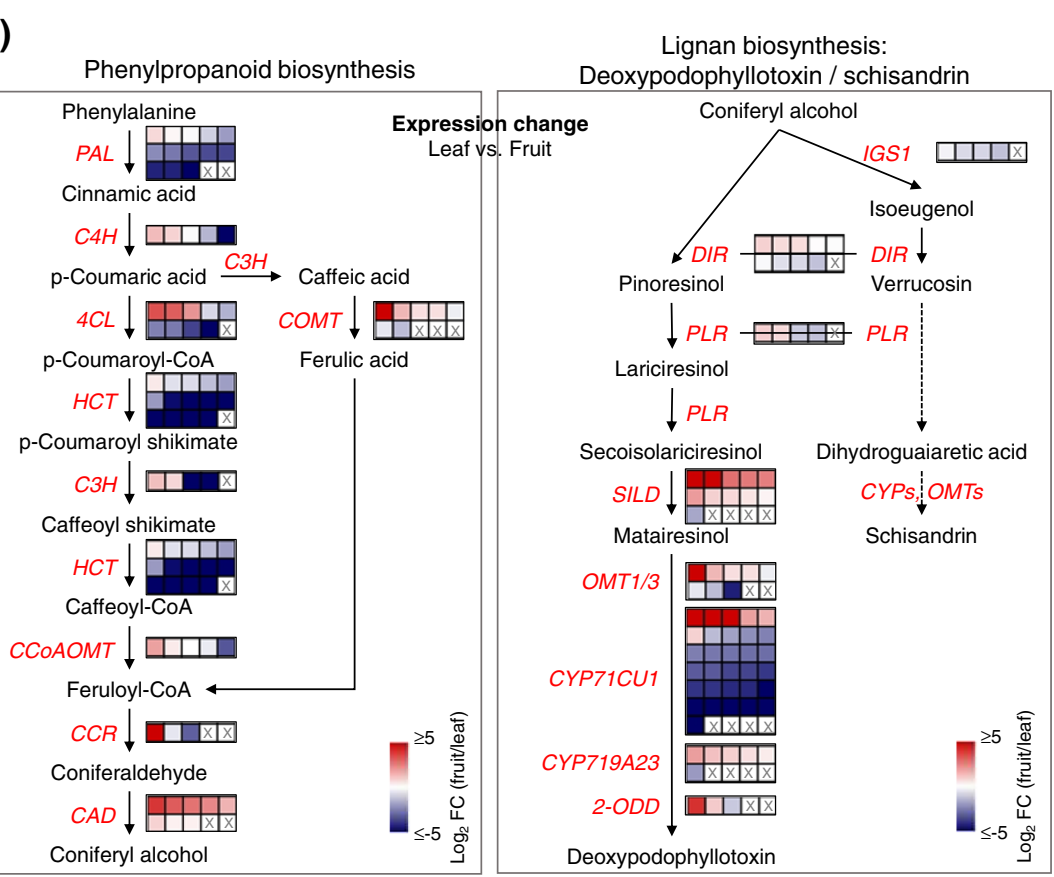

(C)
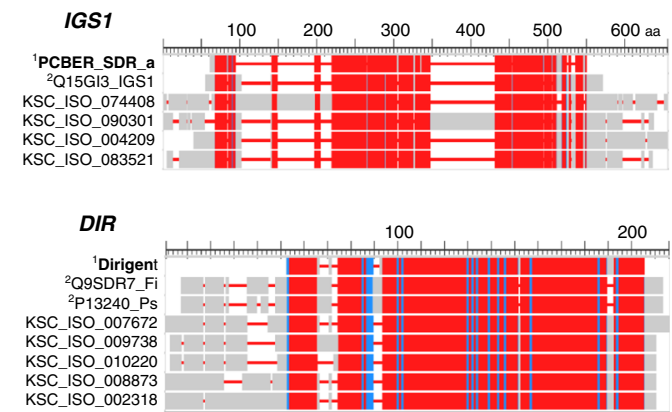

PLR

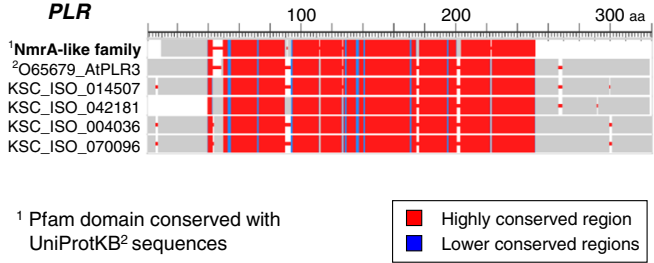

(D)

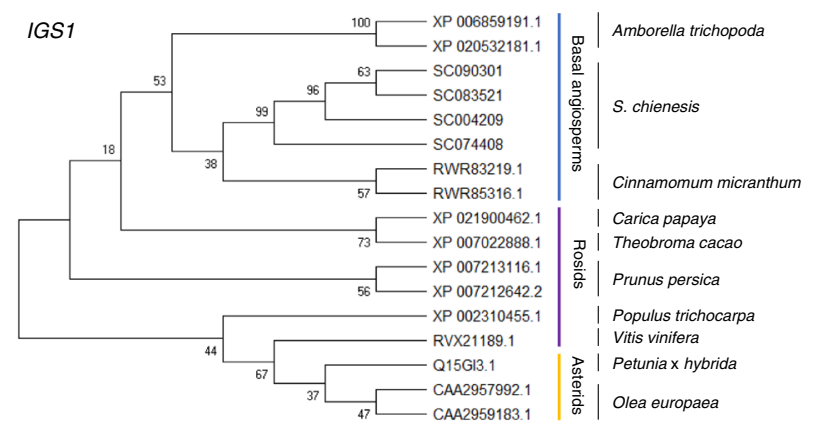

(E)

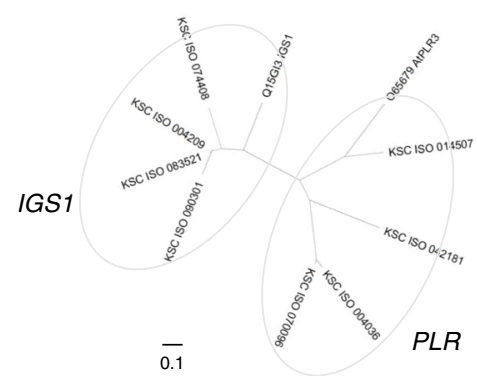

Fig. 3 (See legend on previous page.)

to contain a phenylcoumaran benzylic ether reductase (PCBER)-like protein domain. IGS1 is an atypical shortchain dehydrogenase/reductase (SDR) identified in Petunia hybrid [33] that acts as an NADPH-dependent aromatic alcohol reductase and functions to catalyze the synthesis of phenylpropene isoeugenol from coniferyl alcohol. IGS1 homologs identified in S. chinensis were subordinate to the basal angiosperm group (Fig. 3d) compared with other key lineages that correspond to mostly tree genomes. Second, five DIR unigenes, including KSC_ISO-007672, - 009738, - 010220, - 008873 and-002318, were identified with high sequence 
conservation to the dirigent protein domains of psd_Fi1 and PI206 identified in Forsythia intermedia [34] and Pisum sativum [35]. DIR plays a role in selectively biosynthesizing pinoresinol or verrucosin from two molecules of coniferyl alcohol. Sequence divergences of those DIR unigenes in S. chinensis were identified in their $5^{\prime}$ and $3^{\prime}$ regions. Third, four PLR unigenes, including $\mathrm{KSC}_{-}$ ISO-014507, - 042181, - 004036 and -070096 , were identified with high sequence conservation to AtPLR3 of Arabidopsis, which contains a NmrA-type oxidoreductase family protein domain and might be involved in the reduction of lariciresinol into secoisolariciresinol [10, 36]. Interestingly, although IGS1 and PLR harbored NAD(P)-binding motifs (as members of the SDR family), they showed clear sequence divergence (Fig. 3e). These identified genes are potential candidates for elucidating the regulation of lignan biosynthesis in S. chinensis.

\section{Differential expression of transcripts involved in phenylpropanoid and lignan biosynthesis in the fruit and leaf of S. chinensis}

We also examined the differential expression patterns of transcripts involved in phenylpropanoid and lignan biosynthesis by analyzing RNA-Seq data derived from the deep red-colored fruit and yellow green leaf of S. chinensis cultivar Cheongsoon at 120 days after flowering (DAF) that may lead to the two biosynthesis at the high level. Overall, most unigenes showed divergent expression in the two tissues (Fig. 3b). Transcriptome diversity was even identified among paralogs or isoforms. These results suggest that the tissue-specific expression of transcripts is involved in these two biosynthetic pathways. In phenylpropanoid biosynthesis, most $P A L$ and $H C T$ homologs showed higher expression patterns in the leaves, but all $C A D$ homologs were remarkably upregulated in the fruit (left panel of Fig. 3b), suggesting the predominant production of coniferyl alcohol by CAD in the fruit S. chinensis. Although indirect evidence was obtained, the high content of schisandrin in the fruit of S. chinensis was actually identified by high-performance liquid chromatography (HPLC) profiling (significance at $P<0.01$ ) (Additional file 4: Fig. S3), supporting this hypothesis. In those two pathways for deoxypodophyllotoxin and dihydroguaiaretic acid, three IGS1 unigenes showed slightly high expression in the leaf, but three $D I R$ and two PLR unigenes showed selectively high expression in the fruit with small fold-changes (right panel of Fig. 3b; KSC_ISO-009738 (1.7-fold change) and - 010220 (2.0-fold change) for DIR, and KSC_ISO-014507 (1.8fold change) and-042181 (2.2-fold change) for PLR). Interestingly, most SILD unigenes, which are involved in matairesinol biosynthesis, were upregulated in the fruit, especially KSC_ISO_081641 (71.4-fold change in the fruit) (right panel of Fig. 3b), suggesting the predominant production of matairesinol in the fruit of $S$. chinensis. Our results show the tissue-specific expression of transcripts in the phenylpropanoid and lignan biosynthetic pathways in S. chinensis and their expression diversity.

\section{Transcriptome profiling during fruit development in S. chinensis}

The amounts of dibenzocyclooctadiene lignans in the fruits of S. chinensis are known to be influenced by the degree of fruit maturity and harvest time [3]. To understand transcriptomic changes during fruit development in S. chinensis, we analyzed differentially expressed genes (DEGs) using RNA-Seq data derived from two different fruit development stages in S. chinensis cultivar Cheongsoon, 40 (green-colored and circle-shaped fruit berry) and 120 (deep red-colored and circle-shaped fruit berry) DAF (Fig. 4a), with cutoffs of $q<0.05$, an absolute fold change $\geq 1.5$, and an TPM $\geq 1$ as the minimum average expression value between genes in the two stages. A total of 16,698 DEGs, consisting of 9344 downregulated and 7354 upregulated genes at $120 \mathrm{DAF}$, were identified and subjected to functional categories with Gene Ontology (GO) enrichment analysis. The GO enrichment analysis for genes upregulated at 120 DAF, 904 DEGs, revealed the significance of the functions associated with 'fruit development' $\left(1.39 \times 10^{-12}\right)$, 'Golgi vesicle transport'

\footnotetext{
(See figure on next page.)

Fig. 4 Profile of differentially expressed genes (DEGs) between the green- and red-colored berry stages of S. chinensis. a. Fruit shape at 40 (green-colored berry) and 120 (red-colored fruit berry) days after flowering (DAF) in S. chinensis. b. Gene ontology (GO) enrichment for DEGs between 40 DAF and 120 DAF. The GO enrichment analysis for up- (904 genes) and down- (921 genes) regulated genes at 120 DAF (left panel in Figure) was performed by using DAVID according to an EASE score <0.001, and significant, uniquely represented GO terms were then selected. c. Activation of the phenylpropanoid biosynthetic pathway to coniferyl alcohol at 120 DAF and identification of the corresponding unigenes. $\mathbf{d}$. Mapping to genes in the phenylpropanoid biosynthetic process to other closely related biosynthetic processes, including lignin, suberin, flavonoid, proanthocyanidin, phenol-containing compound, cinnamic acid, karrikin, and cell wall biosynthetic processes. e. Identification of DEGs related to lignan biosynthetic pathways at 40 and 120 DAF. f. Transcriptional network among genes upregulated at 120 DAF related to phenylpropanoid biosynthesis and the responses to ABA, JA, ethylene, and hydrogen peroxide. After selecting up-regulated genes enriched in those five $\mathrm{GO}$ terms, the interaction between those genes was identified by using STRING and further analyzed using Cytoscape on the basis of the degree of connectivity of the nodes. In the network, the interaction between genes in 'phenylpropanoid biosynthetic process' and other functional terms, including 'responses to ABA,',JA mediated signaling,' 'ethylene-activated signaling', or 'response to hydrogen peroxide', are indicated with red-color dot line. In addition, genes in phenylpropanoid pathway are indicated with orange-color letter
} 
(A)

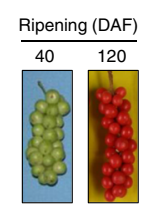

(C)
(B)

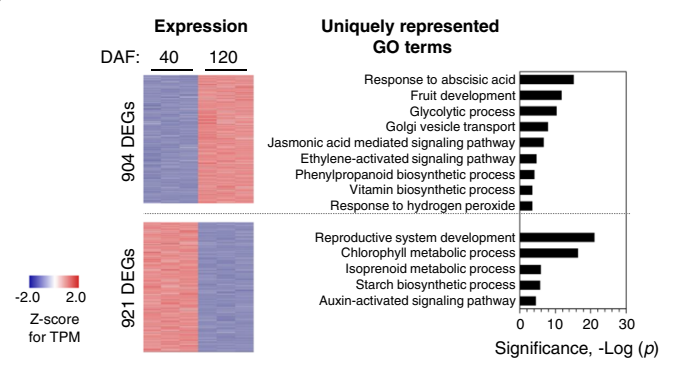

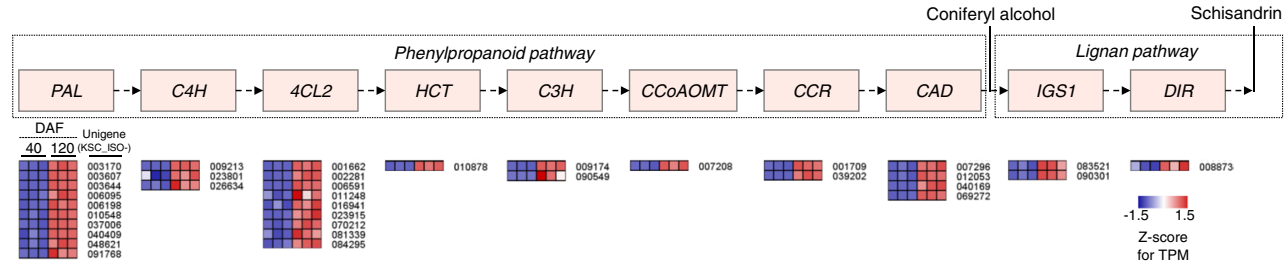

(D)

(E)
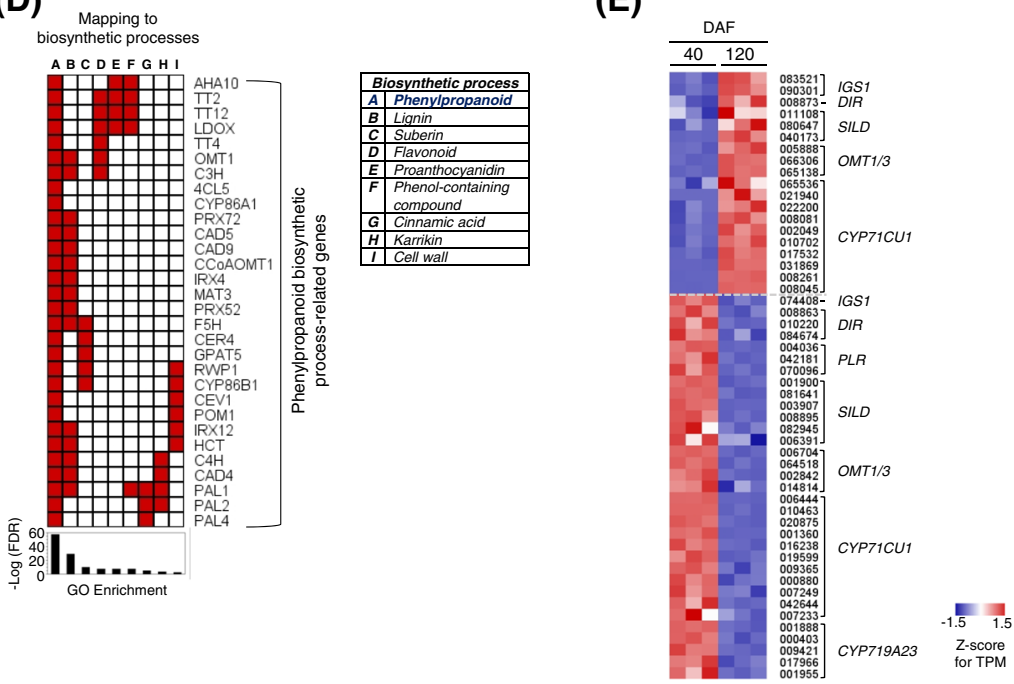

(F)

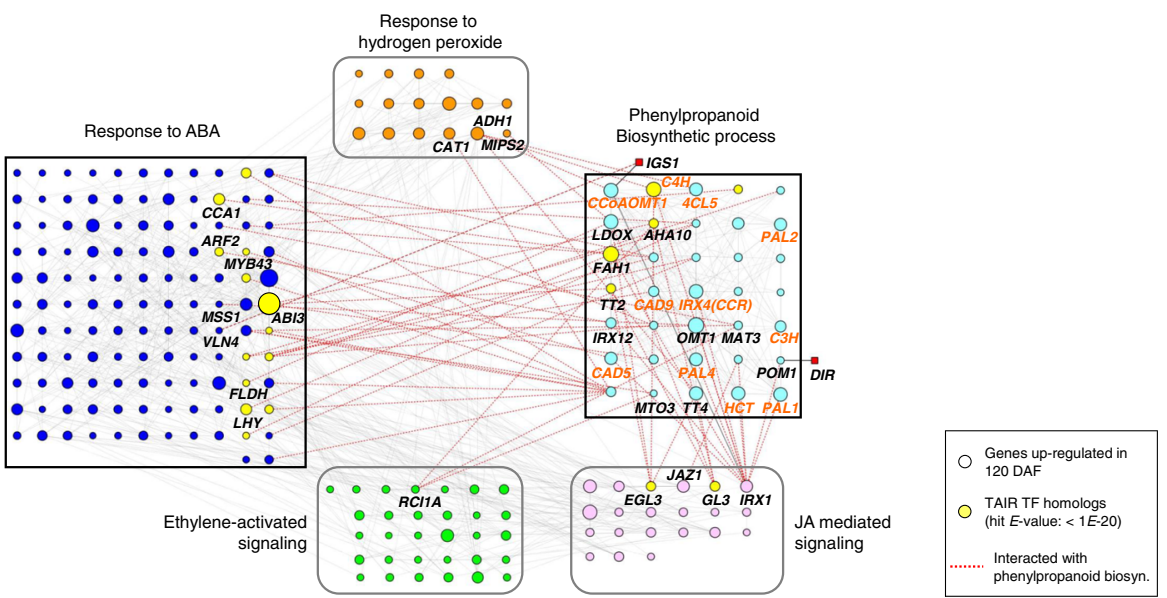

Fig. 4 (See legend on previous page.) 
$\left(1.04 \times 10^{-8}\right)$, 'response to plant hormone signaling pathways' such as abscisic acid (ABA) $\left(4.95 \times 10^{-16}\right)$, ethylene $\left(1.83 \times 10^{-5}\right)$, and jasmonic acid (JA) $\left(1.6 \times 10^{-7}\right)$, 'phenylpropanoid biosynthetic process' $\left(6.87 \times 10^{-5}\right)$, 'vitamin biosynthetic process' $\left(2.69 \times 10^{-4}\right)$, and 'response to hydrogen peroxide $\left(\mathrm{H}_{2} \mathrm{O}_{2}\right)\left(3.34 \times 10^{-4}\right)$ (Fig. 4b). Remarkably, the pathway for coniferyl alcohol production within the phenylpropanoid biosynthetic pathway is likely to be activated as a specific synthesis route of lignan. In this pathway, the expression of $10 P A L, 3 C 4 H, 94 C L 2,1$ $H C T, 2 C 3 H, 1 C C o A O M T, 2 C C R$ and $4 C A D$ unigenes was significantly increased (Fig. 4c). In particular, three CAD homologous unigenes (KSC_ISO-012053, - 040169 and-069272) showed an increase in expression with $\geq 2.7 \sim 10$-fold changes (Additional file 4: Fig. S4). As presented in Additional file 4: Fig. S6, the qRT-PCR data for these DEGs were generally similar with the RNA-seq data, showing a positive correlation $(r=0.601)$. Interestingly, the phenylpropanoid biosynthetic pathway was extensively linked to biosynthetic processes such as those for lignin $\left(\mathrm{FDR}=5.33 \times 10^{-30}\right)$, suberin $\left(3.54 \times 10^{-11}\right)$, proanthocyanidin $\left(1.04 \times 10^{-8}\right)$, flavonoid $\left(8.04 \times 10^{-9}\right)$, phenol-containing compound $\left(2.09 \times 10^{-8}\right)$, cinnamic acid $\left(2.53 \times 10^{-6}\right)$, karrikin $\left(1 \times 10^{-4}\right)$, and cell wall $\left(2.2 \times 10^{-3}\right)$ (Fig. 4 d). This result supports the potential for the activation of other secondary metabolites by the genes involved in the phenylpropanoid biosynthetic pathway at the postfruit development stage of S. chinensis. Therefore, our results indicate the activation of the phenylpropanoid biosynthetic process for lignan biosynthesis at the postfruit development stage of $S$. chinensis. Moreover, genes related to the response to $\mathrm{ABA}$, ethylene-activated signaling, and response to $\mathrm{H}_{2} \mathrm{O}_{2}$, which are known to be associated with fruit ripening [37], are upregulated, implicating a functional link between lignan biosynthesis and ripening. We also identified unigenes upregulated in lignan biosynthetic pathways at 120 DAF (Fig. 4e): 2 IGS1s, 1 DIR, 3 SILDs, 3 OMT1/3, and 10 CYP71CU1. However, unlike IGS1 and DIR unigenes, PLR unigenes showed upregulation at $40 \mathrm{DAF}$. Additionally, most SILD and CYP719A23 unigenes were upregulated at 40 DAF. Collectively, our results show the transcriptional changes in the phenylpropanoid and lignan biosynthetic pathways at postfruit development in S. chinensis.

\section{Transcriptional network between phenylpropanoid biosynthesis and plant hormone signaling}

To investigate the functional links between phenylpropanoid biosynthesis and ripening-related plant hormone signaling in S. chinensis, a transcriptional network of genes upregulated at 120 DAF, which were enriched in the functional categories of 'phenylpropanoid biosynthetic process' and 'responses to plant hormone signaling pathways', including ABA, ethylene, and JA, and 'response to $\mathrm{H}_{2} \mathrm{O}_{2}$, was analyzed. The analysis showed that the phenylpropanoid biosynthetic pathway highly interacted with the ABA and JA signaling pathways (Fig. 4f). In the network, CYP84A1 (FAH1), LDOX, CAD5, TT2, IRX12, $A H A 10$, and OMT1, which are involved in the phenylpropanoid biosynthetic process, interacted with $A B I 3, L H Y$, CCA1, MSS1, FLDH, MYB43, and ARF2 during ABA signaling and $I R X 1, G L 3$, and EGL3 during JA signaling. Three genes, AHA10, MTO3, and MAT3, for the phenylpropanoid biosynthetic process, also interacted with RCI1A during ethylene signaling, which is linked to ABA signaling. In connection with plant hormone signaling, IGS1 and DIR interacted with CCOAOMT1 and POM1 in the phenylpropanoid biosynthetic process, respectively, and IGS1 was linked to VLN4. Additionally, the hydrogen peroxide $\left(\mathrm{H}_{2} \mathrm{O}_{2}\right)$ signaling pathway, including $C A T 1$, $A D H 1$ and MIPS2, also interacted with genes involved in phenylpropanoid biosynthetic processes, including TT4, CCoAOMT1, C4H, and 4CL5. Hydrogen peroxide signaling play an important role in promoting ripening at the molecular level [38]. However, the functions of those three genes associated with fruit ripening remain unknown. These results indicate that the phenylpropanoid biosynthetic pathway is closely connected with the ABA, JA, ethylene, and hydrogen peroxide signaling pathways in the postfruit development stage of S. chinensis and that crosstalk may affect the activation of lignan biosynthesis.

\section{Discussion}

\section{Generation of high-quality reference transcriptome data} for S. chinensis

We generated the de novo transcriptome assembly of S. chinensis cultivar Cheongsoon to obtain insights into the biosynthesis of schisandrin. Because Cheongsoon has the characteristics of high yielding ability and disease tolerance compared with wild type, it has been considered as a plant material for constructing a reference transcriptome in S. chinensis. In addition to the de novo transcriptome assembly of Cheongsoon, we have been working on the project for whole-genome de novo assembly of Cheongsoon. To generate longer unigenes and cover small transcripts in Cheongsoon, a hybrid assembly using long- and short-read sequencing data was employed. This approach showed that the resulting unigene dataset presented a high level of transcript assembly completeness and deep data coverage of the diversity of the transcriptome. Thus, this dataset can lead to the accurate quantification of gene expression and the identification of interesting or novel transcripts of large genes in the absence of whole-genome sequence information [39]. Remarkably, our transcriptome data presented 
considerable unigenes containing complete ORFs with full-length transcripts. This result indicates the potential for effective, accurate gene annotation for elucidating genes associated with traits or functional pathways of interest. For example, a total of 83 full-length unigenes related to phenylpropanoid and lignan biosynthesis in $S$. chinensis could be identified with high homology scores of $<1 E-20$ (Fig. 3), and the conservation of pathways to coniferyl alcohol, deoxypodophyllotoxin, and schisandrin biosynthesis in S. chinensis was identified. In particular, the successful identification of IGS1,DIR, and $P L R$ will be helpful for genome engineering to improve traits associated with pharmacological effects in the fruit of $S$. chinensis. For such reasons, a long-read sequencingbased approach has been recently favored in plants such as red clover, Arabidopsis pumila, grape berry, Taxus cuspidate, and Gossypium austral [40-43], which have not yet undergone whole-genome sequencing. Therefore, our transcriptome data are very useful as reference data for functional genomics and genomic resources for gene prediction and molecular marker development in $S$. chinensis.

\section{Abundance of transcripts related to phenylpropanoid biosynthesis, terpenoid biosynthesis, and gluconeogenesis in S. chinensis transcriptome data}

The functional annotation of the $S$. chinensis transcriptome data revealed the relatively high abundance of transcripts related to phenylpropanoid biosynthesis, terpenoid biosynthesis, and gluconeogenesis. This finding is likely to indicate the predominant activation of those biosynthetic pathways in S. chinensis. In fruit crops, the phenylpropanoid biosynthetic pathway is known to activate the production and accumulation of polyphenols such as lignans and flavonoids [22, 23, 44, 45]. With previous reports, our results provide a clue that explains how lignans such as shisandrins are essentially synthesized by the phenylpropanoid biosynthetic pathway in $S$. chinensis. Interestingly, transcripts related to the pathway exhibited divergent expression in leaves and fruits, implicating functional diversity between different tissues or among isoforms or paralogs. In addition to phenylpropanoid biosynthesis, the abundance of transcripts related to terpenoid biosynthesis was also identified. This result suggests that terpenoids (i.e., wuweizidilactones) in $S$. chinensis might be one of the primary compounds, with lignans having dibenzocyclooctadiene skeletons. The biosynthesis of terpenoids in S. chinensis is not yet known. Some recent studies have reported the identification of new triterpenoids and their bioactivity with weak or no cytotoxicity [46]. We also identified the highest abundance of transcripts related to gluconeogenesis in the transcriptome dataset of $S$. chinensis (Additional file 4:
Fig. S5). Interestingly, gluconeogenesis and the phenylpropanoid biosynthetic pathway are closely linked to fruit ripening processes that lead to the accumulation of sugar and subsequent changes in fruit tissue texture, as well as those that affect phenolic compounds [22, 47]. Moreover, the activation of the glyoxylate cycle, especially the upregulation of malate synthases, during fruit ripening plays an important role in gluconeogenesis via the conversion of lipids to carbohydrates [48-50].

\section{Activation of the phenylpropanoid biosynthetic pathway for lignan biosynthesis at the postfruit development stage of $S$. chinensis and its transcriptional network related to the ABA signaling pathway}

In this study, we identified that the phenylpropanoid biosynthetic pathway tends to be activated for lignan biosynthesis at the postfruit development stage in S. chinensis. This finding was demonstrated by the GO enrichment analysis for genes upregulated in the 120 DAF sample (Fig. 4b). In particular, the analysis revealed that many of genes in the pathway leading to coniferyl alcohol, including PAL, C4H, 4CL2, HCT, C3H, CCoAOMT, CCR and $C A D$, are up-regulated (Fig. 4c). Furthermore, the activation of the pathway is also connected to the upregulation of IGS1 and DIR, which regulate the early steps of schisandrin biosynthesis (Fig. 4c). This result is similar with the finding by Zhang et al. [51]. Koeduka et al. [33] demonstrated that the overexpression of PhIGS1 from petunia induced the accumulation of isoeugenol. Therefore, the up-regulation of genes related to phenylpropanoid biosynthetic pathway at the postfruit development stage seems to elicit the increase of production and accumulation of schisandrin in S. chinensis. In addition to the phenylpropanoid biosynthetic pathway, functions related to the response to $\mathrm{ABA}$, ethylene-activated signaling, and response to $\mathrm{H}_{2} \mathrm{O}_{2}$ were also enriched, with upregulation at the postfruit development stage. These functions that are associated with fruit ripening are closely linked to the activation of the phenylpropanoid biosynthetic pathway and the consequent production and accumulation of lignans $[22,37]$. This result is also supported by our findings that the activated phenylpropanoid biosynthetic pathway highly interacts with fruit ripening-related genes, especially those in the plant hormone signaling pathways of $\mathrm{ABA}$, ethylene, and JA. Interestingly, of those three plant hormone signaling pathways, ABA is likely to be highly associated with lignan biosynthesis. ABA plays a key role in the regulation of PLR expression, which encodes a pinoresinol lariciresinol reductase, and lignan accumulation in Linum usitatissimum, which requires two cis-acting elements, $A B A$ response element $(A B R E)$ and $M Y B 2$, for this regulation [52]. The motif matrices of transcription factor ABI3 has significant affinity for the 
DIR3 promoter with seed-specific expression [53]. MYB transcription factors MYB43, MYB20, and MYB85 are transcriptional regulators that directly activate lignin and phenylalanine biosynthesis genes during secondary wall formation in Arabidopsis [54]. Additionally, in relation to JA, the bHLH transcription factor EGL3 and its close homolog GL3 are important regulators of the anthocyanin pathway in Arabidopsis thaliana [55]. Collectively, our results suggest that the activation of phenylpropanoid biosynthesis at the postfruit development stage is involved in the regulation of IGS1 in lignan biosynthetic pathway. Moreover, genes related to ABA hormone signaling, especially ABI and MYB transcription factors, that is associated with fruit ripening and highly interact with phenylpropanoid biosynthetic pathway are also likely to be involved in the regulation of DIR and PLR in lignan pathway, thus contributing to the production and accumulation of schisandrin.

\section{Conclusions}

We generated a longer, high-quality unigene dataset to obtain a more comprehensive view of lignan biosynthesis in S. chinensis. These data can be effectively utilized as reference data for the functional genomics of S. chinensis. Our transcriptome data cover transcripts for elucidating lignan and its precursor biosynthetic pathways. Remarkably, this study suggests that the activation of the phenylpropanoid biosynthetic pathway, along with the formation of a transcriptional network with fruit ripening-related genes, at the postfruit development stage of $S$. chinensis is highly linked to the production and accumulation of schisandrin. Therefore, our results will provide insight into lignan biosynthesis during fruit development in S. chinensis.

\section{Materials and methods}

\section{Plant sampling and RNA preparation}

Three tissues, leaves, roots, and fruits, from the S. chinensis cultivar Cheongsoon were collected (Additional file 1: Table S1; Additional file 2: Table S2). The cultivar Cheongsoon and a wild type Sobaeksan has been grown in the natural environment at the Medicinal Herb Resource Research Institute at Jeonbuk Agricultural Research \& Extension Services (JABARES) in Jinan, Republic of Korea. The voucher specimens for Cheongsoon (MJ3-7) and Sobaeksan (SB-1) were deposited at the herbarium of the Medicinal Herb Resource Research Institute at JABARES. All of the tissues were cut into small pieces and frozen in liquid nitrogen, and $\geq 1 \mu \mathrm{g}$ of total RNA from each sample was then extracted using an easy spin RNA extraction kit (iNtRON Biotechnology) according to the manufacturer's instructions. The quality of the total RNA was assessed on an Agilent 2100 Bioanalyzer (Agilent Technologies, Santa Clara, CA) according to the criteria of an RNA integrity number (RIN) of 7.1 and a $28 \mathrm{~S} / 18 \mathrm{~S}$ ratio of 1.0 on average.

\section{Iso-Seq and RNA-Seq}

For PacBio Iso-Seq, one microgram of an RNA mixture obtained from equal amounts of RNA from the three tissues of Cheongsoon was reverse transcribed using the Clontech SMARTer cDNA synthesis kit (Clontech Laboratories, CA, USA). cDNA synthesis was followed by size selection according to length categories of $1-2 \mathrm{~kb}$, $2-3 \mathrm{~kb}, 3-6 \mathrm{~kb}$, and $>6 \mathrm{~kb}$ using BluePippin (Sage Science, Beverly, MA). The products were purified using AMPure PB beads (Pacific Biosciences, USA). The amplified and size-selected cDNA products were employed to generate SMRTbell template libraries according to the PacBio Iso-Seq protocol (Pacific Biosciences, Menlo Park, CA, USA). The libraries were prepared for sequencing via the annealing of a sequencing primer (component of the SMRTbell Template Prep Kit 1.0), followed by polymerase binding to the primer-annealed template. SMRT cells were sequenced on the PacBio RS II platform (Pacific Biosciences, Menlo Park, CA, USA) using P6-C4 chemistry with $3 \sim 4$ h movies (Additional file 1: Table S1).

For Illumina RNA-Seq analysis, RNA-Seq libraries were prepared from $1 \mu \mathrm{g}$ of total RNA derived from fruit and leaf using a TruSeq RNA Sample Prep Kit according to the manufacturer's manual (Illumina, Inc., San Diego, CA). cDNA was synthesized from the mRNA fragments and was then subjected to an end repair process, the addition of a single 'A' base, and the ligation of the adapters. The libraries were purified and enriched via PCR amplification and were then subjected to pairedend sequencing with a $100 \mathrm{bp}$ read length on the Illumina HiSeq 4000 platform (Additional file 2: Table S2).

\section{De novo transcript assembly}

Iso-Seq raw reads were processed with PacBio SMRTlink (version 8.0) (https://www.pacb.com/support/softwaredownloads). First, reads of inserts (ROIs) were generated according to a minimum read quality of 75 . ROIs were classified into full-length nonchimeric and non-full-length reads by identifying the $5^{\prime}$ and $3^{\prime}$ adapters employed in library preparation using Classify. Full-length reads were defined as those containing both adapters. All of the fulllength reads were clustered using Cluster, and the clustered consensus sequences were then polished together with the non-full-length reads using Quiver. The high- and low-quality consensus sequences were clustered once more using CD-HIT (version 4.6.6) [56] with a sequence identity threshold of 0.99. De novo assembly using RNA-Seq was 
conducted with Trinity (version 2.0.4) [57]. After assembly, nonredundant representative sequences (referred to as unigenes) were generated by using the TGI Clustering Tool (TGICL) [58] and CD-HIT (version 4.6.6) [56]. After de novo assembly, unigenes belonging to TE sequences that were screened using RepeatMasker (version 4.0.6) (http:// repeatmasker.org) were removed. The unigenes resulting from the Iso-Seq and RNA-Seq assemblies were merged. In the final unigene dataset, protein coding sequences (CDSs) were analyzed by using TransDecoder (version 3.0.0) [59] with the following steps: (1) a search for all possible CDSs, (2) verification of CDSs using GeneID [60], and (3) selection of the region with the highest score. To assess the quality of the assembled transcripts (or unigenes), CEGMA (version 2.5) [61], which assesses a highly reliable set of gene annotations during genome and transcriptome assembly, was employed together with the Eukaryotic Orthologous Groups of proteins (KOG) database. Moreover, the compatibility of unigenes with other datasets was analyzed by mapping with BWA (version 0.7.10) [62] using RNA-Seq samples derived from leaf and fruit tissues with the following parameters: $-\mathrm{k} 19$ for the minimum seed length, $-\mathrm{A} 1$ for the matching score, $-\mathrm{B} 4$ for the mismatch penalty, and $-\mathrm{T} 30$ for the alignment output with a score higher than 30 .

\section{Functional annotation of unigenes}

Unigenes were searched against the UniProt, NCBI nonredundant (NR), TAIR, and PlantTFDB databases using BLASTX (version 2.2.29+) [63] with a cutoff $E$-value of $1 E-5$. Protein domains were also searched using InterProScan (version 5.17-56.0) [64]. GO and Kyoto Encyclopedia of Genes and Genomes (KEGG) pathway annotations were performed using Blast2GO (version 5.2.5) $[28,29,65]$. To identify candidate genes, such as DIR, IGS1, and PLR, first, the homologous unigenes were searched against UniProt, TAIR, and Plant Metabolic Pathway (https://plantcyc.org) databases using BLASTX with a cutoff of $E$-value $<1 E-20$. Then, these unigenes were curated based on their protein domain data by using InterProScan, compared with corresponding conserved protein domains via multiple-sequence alignment using MUSCLE (3.8.31) [66], and further analyzed by using COBALT [67] to visualize the conservation among sequences (https://www.ncbi.nlm.nih.gov/tools/cobalt). Phylogenetic analysis was performed using MEGA-X via the maximum likelihood-based method [68].

\section{Differential expression analysis}

To examine differential expression patterns between redcolored fruit and yellow green leaf at 120 DAF for tissuespecific expression of transcripts, and between fruits collected at 40 (green-colored fruit berries) and 120 (deep red-colored fruit berries) DAF for transcriptome profiling during fruit development, respectively, RNA-Seq data derived from those four samples of Cheongsoon were analyzed. Clean RNA-Seq reads were mapped to the unigenes of S. chinensis using Bowtie 2 (version 2.4.4) [69]. Transcript abundance was estimated using RSEM (version 1.2.19) [70] as transcripts per million (TPM) values. Based on the resulting quantification results, the expression differences between fruit and leaf samples at $120 \mathrm{DAF}$, and between fruit samples collected at 40 and 120 DAF were analyzed using the TCC package (version 1.28.0) [71], respectively, with cutoffs of $q<0.05$, an absolute fold-change $\geq 1.5$, and an $\mathrm{TPM} \geq 1$ as the minimum average expression value between genes identified in the two samples. DEGs between fruit samples collected at 40 and 120 DAF were further annotated. GO enrichment analysis for DEGs that were specifically annotated with known genes in TAIR was performed by using DAVID (version 6.8) [72] according to an EASE score $<0.001$. Genes categorized with GO-enriched terms were selected, and their expression patterns were visualized in a heatmap by using $\mathrm{MeV}$ (http://mev.tm4.org) with the Euclidean distance and complete linkage method. To investigate protein-protein interactions, genes categorized with specific GO terms were searched against STRING [73] with medium confidence $(\geq 0.4)$. The network was further analyzed using Cytoscape (www.cytoscape.org).

\section{Abbreviations \\ ABA: Abscisic acid; CEGMA: Core Eukaryotic Genes Mapping Approach; DAF: Day after flowering; DEG: Differentially expressed gene; DIR: Dirigent-like protein; EGS1: Eugenol synthase 1; GO: Gene ontology; HPLC: High-perfor- mance liquid chromatography; IGS1: Isoeugenol synthase 1; Iso-Seq: Isoform sequencing; JA: Jasmonic acid; KEGG: Kyoto Encyclopedia of Genes and Genomes; ORF: Open reading frame; PLR: Pinoresinol-lariciresinol reductase; RNA-Seq: RNA sequencing; SDR: Short-chain dehydrogenase/reductase; TE: Transposable element; TF: Transcription factor.}

\section{Supplementary Information}

The online version contains supplementary material available at https://doi. org/10.1186/s12864-021-08253-2.

Additional file 1: Table S1. Summary of subreads generated by Iso-Seq. Additional file 2: Table S2. Summary of RNA-Seq in S. chinensis.

Additional file 3: Table S3. Summary for circular consensus sequencing (CCS) reads.

Additional file 4: Figure S1-S7

Additional file 5: Table S4. Phenotypic characteristics of S. chinensis samples used in the study.

Additional file 6: Table S5. Primers used for QRT-PCR validation.

\section{Acknowledgements}

We thank to CEO Samuel Hwang (Theragen Bio Co., Ltd., South Korea) supporting computing system for bioinformatics analyses.

\section{Authors' contributions}

$\mathrm{CPH}$ and SHK designed and supervised the study; CKK, HJJ, YL, and HJK collected samples; CKK, JNK, SJK, and SHK carried out transcriptome sequencing; 
HJJ and YL carried out HPLC; CPH, DJL, SGP carried out bioinformatics analyses; $\mathrm{CPH}, \mathrm{SHK}$, and HR wrote the manuscript. All authors read and approved the final manuscript.

\section{Funding}

This work was supported by the Cooperative Research Program for National Agricultural Genome Program (Grant No. PJ01349002), Rural Development Administration (RDA), Republic of Korea.

\section{Availability of data and materials}

Sequencing data used in this study are available in the NCBI Sequence Read Archive (SRA) database under the following accession numbers: SAMN19471228 for PacBio Iso-Seq data (https://www.ncbi.nlm.nih.gov/biosa mple/SAMN19471228) and SAMN19471229 - SAMN19471233 (https://www. ncbi.nlm.nih.gov/biosample/SAMN19471229/; https://www.ncbi.nlm.nih.gov/ biosample/SAMN19471230; https://www.ncbi.n/m.nih.gov/biosample/SAMN1 9471231; https://www.ncbi.nIm.nih.gov/biosample/SAMN19471232; https:// www.ncbi.nlm.nih.gov/biosample/SAMN19471233) for Illumina RNA-Seq.

\section{Declarations}

\section{Ethics approval and consent to participate}

Schisandra chinensis cultivar 'Cheongsoon' and wild type 'Sobaeksan' were collected from the Medicinal Herb Resource Research Institute at Jeonbuk Agricultural Research \& Extension Services (JABARES) in Jinan, Republic of Korea with permission from the JABARES researcher, Mr. Hyo-Jin Kim. The plants are freely accessible to Hyo-Jin Kim with non-commercial resource purpose. The authors comply with relevant institutional, national, and international guidelines and legislation for plant study.

\section{Consent for publication}

Not applicable.

\section{Competing interests}

The authors declare that no competing interests exist.

\section{Author details}

${ }^{1}$ Theragen Bio Co., Ltd., Suwon 16229, Republic of Korea. ${ }^{2}$ Genomics Division, National Institute of Agricultural Sciences, RDA, Jeonju 54874, Republic of Korea. ${ }^{3}$ Department of Industrial Plant Science \& Technology, Chungbuk National University, Cheongju 28644, Republic of Korea. ${ }^{4}$ Jeollabukdo ARES Medicinal Resource Research Institute, Jinan 55440, Republic of Korea. ${ }^{5}$ Department of Biological Sciences and Biotechnology, Chungbuk National University, Cheongju 28644, Republic of Korea.

\section{Received: 21 June 2021 Accepted: 14 December 2021}

Published online: 08 January 2022

\section{References}

1. Li B, Zheng Y. Dynamic evolution and phylogenomic analysis of the chloroplast genome in Schisandraceae. Sci Rep. 2018;8(1):9285.

2. Ye JW, Zhang ZK, Wang HF, Bao L, Ge JP. Phylogeography of Schisandra chinensis (Magnoliaceae) reveal multiple refugia with ample gene flow in Northeast China. Front Plant Sci. 2019;10:199.

3. Szopa A, Ekiert R, Ekiert H. Current knowledge of Schisandra chinensis (Turcz.) Baill. (Chinese magnolia vine) as a medicinal plant species: a review on the bioactive components, pharmacological properties, analytical and biotechnological studies. Phytochem Rev. 2017;16(2):195-218.

4. Nowak A, Zaklos-Szyda M, Blasiak J, Nowak A, Zhang Z, Zhang B. Potential of Schisandra chinensis (Turcz.) Baill. In human health and nutrition: a review of current knowledge and therapeutic perspectives. Nutrients. 2019;11(2):333.

5. Park JY, Yun JW, Choi YW, Bae JU, Seo KW, Lee SJ, et al. Antihypertensive effect of gomisin a from Schisandra chinensis on angiotensin II-induced hypertension via preservation of nitric oxide bioavailability. Hypertens Res. 2012;35(9):928-34.

6. Szopa A, Dziurka M, Warzecha A, Kubica P, Klimek-Szczykutowicz M, Ekiert $H$. Targeted lignan profiling and anti-inflammatory properties of Schisandra rubriflora and Schisandra chinensis extracts. Molecules. 2018;23(12):3103.
7. Kim JS, Takanche JS, Kim JE, Jeong SH, Han SH, Yi HK. Schisandra chinensis extract ameliorates age-related muscle wasting and bone loss in ovariectomized rats. Phytother Res. 2019;33(7):1865-77.

8. Sowndhararajan K, Deepa P, Kim M, Park SJ, Kim S. An overview of neuroprotective and cognitive enhancement properties of lignans from Schisandra chinensis. Biomed Pharmacother. 2018;97:958-68.

9. Davin LB, Lewis NG. An historical perspective on lignan biosynthesis: Monolignol, allylphenol and hydroxycinnamic acid coupling and downstream metabolism. Phytochem Rev. 2003;2:257.

10. Dinkova-Kostova AT, Gang DR, Davin LB, Bedgar DL, Chu A, Lewis NG. $(+)$-Pinoresinol/(+)-lariciresinol reductase from Forsythia intermedia. Protein purification, cDNA cloning, heterologous expression and comparison to isoflavone reductase. J Biol Chem. 1996;271:29473-82.

11. Hemmati S, Schmidt TJ, Fuss E. (+)-Pinoresinol/(-)-lariciresinol reductase from Linum perenne Himmelszelt involved in the biosynthesis of justicidin B. FEBS Lett. 2007;581(4):603-10.

12. Nakatsubo T, Mizutani M, Suzuki S, Hattori T, Umezawa T. Characterization of Arabidopsis thaliana pinoresinol reductase, a new type of enzyme involved in lignan biosynthesis. J Biol Chem. 2008:283(23):15550-7.

13. Xia ZQ, Costa MA, Pelissier HC, Davin LB, Lewis NG. Secoisolariciresinol dehydrogenase purification, cloning, and functional expression. Implications for human health protection. J Biol Chem. 2001;276(16):12614-23.

14. Lau W, Sattely ES. Six enzymes from mayapple that complete the biosynthetic pathway to the etoposide aglycone. Science. 2015;349(6253):1224-8.

15. Satake H, Koyama T, Bahabadi SE, Matsumoto E, Ono E, Murata J. Essences in metabolic engineering of lignan biosynthesis. Metabolites. 2015;5(2):270-90.

16. Kim HJ, Ono E, Morimoto K, Yamagaki T, Okazawa A, Kobayashi A, et al. Metabolic engineering of lignan biosynthesis in Forsythia cell culture. Plant Cell Physiol. 2009;50(12):2200-9.

17. Umezawa T. Diversity in lignan biosynthesis. Phytochem Rev. 2003;2:371-90.

18. Tulipani S, Marzban G, Herndl A, Laimer M, Mezzetti B, Battino M. Influence of environmental and genetic factors on health-related compounds in strawberry. Food Chem. 2011;124(3):906-13.

19. Elmastas M, Demir A, Genc N, Dolek U, Gunes M. Changes in flavonoid and phenolic acid contents in some Rosa species during ripening. Food Chem. 2017;235:154-9.

20. Zhang H, Zhang GQ, Zhu ZY, Zhao L, Fei Y, Jing J, et al. Determination of six lignans in Schisandra chinensis (Turcz.) Baill. Fruits and related Chinese multiherb remedies by HPLC. Food Chem. 2009;115(2):735-9.

21. Liu HT, Lai HW, Jia XY, Liu JS, Zhang Z, Qi YD, et al. Comprehensive chemical analysis of Schisandra chinensis by HPLC-DAD-MS combined with chemometrics. Phytomedicine. 2013;20(12):1135-43.

22. Singh R, Rastogi S, Dwivedi UN. Phenylpropanoid metabolism in ripening fruits. Compr Rev Food Sci Food Saf. 2010;9(4):398-416.

23. Sharma A, Shahzad B, Rehman A, Bhardwaj R, Landi M, Zheng B. Response of Phenylpropanoid pathway and the role of polyphenols in plants under abiotic stress. Molecules. 2019;24(13):2452.

24. Jo IH, Lee J, Hong CE, Lee DJ, Bae W, Park SG, et al. Isoform sequencing provides a more comprehensive view of the panax ginseng transcriptome. Genes-Basel. 2017;8(9):228.

25. Wang B, Tseng E, Regulski M, Clark TA, Hon T, Jiao Y, et al. Unveiling the complexity of the maize transcriptome by single-molecule long-read sequencing. Nat Commun. 2016;7:11708.

26. Hu Z, Zhang Y, He Y, Cao Q, Zhang T, Lou L, et al. Full-length transcriptome assembly of italian ryegrass root integrated with RNA-seq to identify genes in response to plant cadmium stress. Int J Mol Sci. 2020;21(3):1067.

27. Chen CY, Liu SY, Yan Y, Yin L, Di P, Liu HM, et al. Candidate genes involved in the biosynthesis of lignan in Schisandra chinensis fruit based on transcriptome and metabolomes analysis. Chin J Nat Med. 2020;18(9):684-95.

28. Kanehisa M, Goto S. KEGG: Kyoto encyclopedia of genes and genomes. Nucleic Acids Res. 2000;28(1):27-30.

29. Kanehisa M, Furumichi M, Sato Y, Ishiguro-Watanabe M, Tanabe M. KEGG: integrating viruses and cellular organisms. Nucleic Acids Res. 2021:49(D1):D545-51.

30. Bianchi VJ, Rubio M, Trainotti L, Verde I, Bonghi C, Martinez-Gomez P. Prunus transcription factors: breeding perspectives. Front Plant Sci. 2015:6:443. 
31. Suzuki S, Umezawa T. Biosynthesis of lignans and norlignans. J Wood Sci. 2007:53:273-84.

32. Amborella Genome P. The Amborella genome and the evolution of flowering plants. Science. 2013;342(6165):1241089.

33. Koeduka T, Fridman E, Gang DR, Vassao DG, Jackson BL, Kish CM, et al. Eugenol and isoeugenol, characteristic aromatic constituents of spices, are biosynthesized via reduction of a coniferyl alcohol ester. Proc Natl Acad Sci U S A. 2006;103(26):10128-33.

34. Gang DR, Costa MA, Fujita M, Dinkova-Kostova AT, Wang HB, Burlat V, et al. Regiochemical control of monolignol radical coupling: a new paradigm for lignin and lignan biosynthesis. Chem Biol. 1999;6(3):143-51.

35. Culley DE, Horovitz D, Hadwiger LA. Molecular characterization of disease-resistance response gene DRR206-d from Pisum sativum (L.). Plant Physiol. 1995;107(1):301-2.

36. Gang DR, Kasahara H, Xia ZQ, Vander Mijnsbrugge K, Bauw G, Boerjan $W$, et al. Evolution of plant defense mechanisms. Relationships of phenylcoumaran benzylic ether reductases to pinoresinol-lariciresinol and isoflavone reductases. J Biol Chem. 1999:274(11):7516-27.

37. Kumar R, Khurana A, Sharma AK. Role of plant hormones and their interplay in development and ripening of fleshy fruits. J Exp Bot. 2014;65(16):4561-75.

38. Guo DL, Wang ZG, Pei MS, Guo LL, Yu YH. Transcriptome analysis reveals mechanism of early ripening in Kyoho grape with hydrogen peroxide treatment. BMC Genomics. 2020;21(1):784.

39. Uapinyoying P, Goecks J, Knoblach SM, Panchapakesan K, Bonnemann CG, Partridge TA, et al. A long-read RNA-seq approach to identify novel transcripts of very large genes. Genome Res. 2020;30(6):885-97.

40. Minio A, Massonnet M, Figueroa-Balderas R, Vondras AM, Blanco-Ulate B, Cantu D. Iso-Seq allows Genome-independent transcriptome profiling of grape berry development. G3 (Bethesda). 2019;9(3):755-67.

41. Yang L, Jin Y, Huang W, Sun Q, Liu F, Huang X. Full-length transcriptome sequences of ephemeral plant Arabidopsis pumila provides insight into gene expression dynamics during continuous salt stress. BMC Genomics. 2018;19(1):717.

42. Chao Y, Yuan J, Li S, Jia S, Han L, Xu L. Analysis of transcripts and splice isoforms in red clover (Trifolium pratense L.) by single-molecule long-read sequencing. BMC Plant Biol. 2018;18(1):300.

43. Kuang $X$, Sun S, Wei J, Li Y, Sun C. Iso-Seq analysis of the Taxus cuspidata transcriptome reveals the complexity of Taxol biosynthesis. BMC Plant Biol. 2019;19(1):210.

44. Hoffmann T, Kalinowski G, Schwab W. RNAi-induced silencing of gene expression in strawberry fruit (Fragaria $x$ ananassa) by agroinfiltration: a rapid assay for gene function analysis. Plant J. 2006;48(5):818-26.

45. Hoffmann T, Kurtzer R, Skowranek K, Kiessling P, Fridman E, Pichersky $E$, et al. Metabolic engineering in strawberry fruit uncovers a dormant biosynthetic pathway. Metab Eng. 2011;13(5):527-31.

46. Liu GZ, Liu Y, Sun YP, Li XM, Xu ZP, Jiang P, et al. Lignans and Terpenoids from the leaves of Schisandra chinensis. Chem Biodivers. 2020;17(4):e2000035.

47. Quinet M, Angosto T, Yuste-Lisbona FJ, Blanchard-Gros R, Bigot S, Martinez JP, et al. Tomato fruit development and metabolism. Front Plant Sci. 2019:10:1554

48. Eastmond PJ, Graham IA. Re-examining the role of the glyoxylate cycle in oilseeds. Trends Plant Sci. 2001;6(2):72-8

49. Pua EC, Chandramouli S, Han P, Liu P. Malate synthase gene expression during fruit ripening of Cavendish banana (Musa acuminata cv. Williams), J Exp Bot. 2003;54(381):309-16.

50. Centeno DC, Osorio S, Nunes-Nesi A, Bertolo AL, Carneiro RT, Araujo $W L$, et al. Malate plays a crucial role in starch metabolism, ripening, and soluble solid content of tomato fruit and affects postharvest softening. Plant Cell. 2011;23(1):162-84.

51. Zhang L, Chen J, Zhou X, Chen X, Li Q, Tan H, et al. Dynamic metabolic and transcriptomic profiling of methyl jasmonate-treated hairy roots reveals synthetic characters and regulators of lignan biosynthesis in Isatis indigotica fort. Plant Biotechnol J. 2016;14(12):2217-27.

52. Markulin L, Drouet S, Corbin C, Decourtil C, Garros L, Renouard S, et al. The control exerted by ABA on lignan biosynthesis in flax (Linum usitatissimum $\mathrm{L}$.) is modulated by a $\mathrm{Ca}(2+)$ signal transduction involving the calmodulin-like LuCML15b. J Plant Physiol. 2019;236:74-87.

53. Paniagua C, Bilkova A, Jackson P, Dabravolski S, Riber W, Didi V, et al. Dirigent proteins in plants: modulating cell wall metabolism during abiotic and biotic stress exposure. J Exp Bot. 2017;68(13):3287-301 .
54. Geng P, Zhang S, Liu J, Zhao C, Wu J, Cao Y, et al. MYB20, MYB42, MYB43, and MYB85 regulate phenylalanine and lignin biosynthesis during secondary Cell Wall formation. Plant Physiol. 2020;182(3):1272-83.

55. Feyissa DN, Lovdal T, Olsen KM, Slimestad R, Lillo C. The endogenous $G L 3$, but not $E G L 3$, gene is necessary for anthocyanin accumulation as induced by nitrogen depletion in Arabidopsis rosette stage leaves. Planta. 2009;230(4):747-54.

56. Fu L, Niu B, Zhu Z, Wu S, LiW. CD-HIT: accelerated for clustering the nextgeneration sequencing data. Bioinformatics. 2012;28(23):3150-2.

57. Grabherr MG, Haas BJ, Yassour M, Levin JZ, Thompson DA, Amit I, et al. Full-length transcriptome assembly from RNA-Seq data without a reference genome. Nat Biotechnol. 2011;29(7):644-52.

58. Pertea G, Huang X, Liang F, Antonescu V, Sultana R, Karamycheva S, et al. TIGR gene indices clustering tools (TGICL): a software system for fast clustering of large EST datasets. Bioinformatics. 2003;19(5):651-2.

59. Haas BJ, Papanicolaou A, Yassour M, Grabherr M, Blood PD, Bowden J, et al. De novo transcript sequence reconstruction from RNA-seq using the trinity platform for reference generation and analysis. Nat Protoc. 2013:8(8):1494-512.

60. Blanco E, Parra G, Guigó R. Using Geneid to identify genes. Hoboken, NJ, USA: John Wiley \& Sons, Inc.; 2007.

61. Parra G, Bradnam K, Korf I. CEGMA: a pipeline to accurately annotate core genes in eukaryotic genomes. Bioinformatics. 2007;23(9):1061-7.

62. Li H, Durbin R. Fast and accurate short read alignment with burrowswheeler transform. Bioinformatics. 2009:25(14):1754-60.

63. Altschul SF, Gish W, Miller W, Myers EW, Lipman DJ. Basic local alignment search tool. J Mol Biol. 1990;215(3):403-10.

64. Zdobnov EM, Apweiler R. InterProScan--An integration platform for the signature-recognition methods in InterPro. Bioinformatics. 2001;17(9):847-8.

65. Conesa A, Gotz S, Garcia-Gomez JM, Terol J, Talon M, Robles M. Blast2GO: a universal tool for annotation, visualization and analysis in functional genomics research. Bioinformatics. 2005;21 (18):3674-6.

66. Edgar RC. MUSCLE: multiple sequence alignment with high accuracy and high throughput. Nucleic Acids Res. 2004;32(5):1792-7.

67. Papadopoulos JS, Agarwala R. COBALT: constraint-based alignment tool for multiple protein sequences. Bioinformatics. 2007;23(9):1073-9.

68. Kumar S, Stecher G, Li M, Knyaz C, Tamura K. MEGA X: molecular evolutionary genetics analysis across computing platforms. Mol Biol Evol. 2018;35(6):1547-9.

69. Langmead B, Salzberg SL. Fast gapped-read alignment with bowtie 2. Nat Methods. 2012;9(4):357-9.

70. Li B, Dewey CN. RSEM: accurate transcript quantification from RNASeq data with or without a reference genome. BMC Bioinformatics. 2011;12:323.

71. Sun J, Nishiyama T, Shimizu K, Kadota K. TCC: an R package for comparing tag count data with robust normalization strategies. BMC Bioinformatics. 2013;14:219.

72. Huang DW, Sherman BT, Tan Q, Collins JR, Alvord WG, Roayaei J, et al. The DAVID gene functional classification tool: a novel biological modulecentric algorithm to functionally analyze large gene lists. Genome Biol. 2007;8(9):R183.

73. Szklarczyk D, Gable AL, Lyon D, Junge A, Wyder S, Huerta-Cepas J, et al. STRING v11: protein-protein association networks with increased coverage, supporting functional discovery in genome-wide experimental datasets. Nucleic Acids Res. 2019;47(D1):D607-13.

\section{Publisher's Note}

Springer Nature remains neutral with regard to jurisdictional claims in published maps and institutional affiliations. 